\title{
The Riemann Hypothesis-Millennium Prize Problem
}

\section{A. A. Durmagambetov}

Karaganda State University, Karagandy, Kazakhstan

Email: aset.durmagambet@gmail.com

How to cite this paper: Durmagambetov, A.A. (2016) The Riemann Hypothesis-Millennium Prize Problem. Advances in Pure Mathematics, 6, 915-920.

http://dx.doi.org/10.4236/apm.2016.612069

Received: October 3, 2016

Accepted: November 19, 2016

Published: November 22, 2016

Copyright $\odot 2016$ by author and Scientific Research Publishing Inc. This work is licensed under the Creative Commons Attribution International License (CC BY 4.0).

http://creativecommons.org/licenses/by/4.0/

\begin{abstract}
This work is dedicated to the promotion of the results C. Muntz obtained modifying zeta functions. The properties of zeta functions are studied; these properties lead to new regularities of zeta functions. The choice of a special type of modified zeta functions allows estimating the Riemann's zeta function and solving Riemann ProblemMillennium Prize Problem.
\end{abstract}

\section{Keywords}

Euler, Chebyshev, Dirichlet, Riemann, Hypothesis, Zeta Function, Muntz, Function, Complex Numbers, Regular Function, Integral Function Representation, Millennium Prize Problem

\section{(c) (i) Open Access}

\section{Introduction}

In this work we are studying the properties of modified zeta functions. Riemann's zeta function is defined by the Dirichlet's distribution

$$
\varsigma(z)=\sum_{n=1}^{\infty} \frac{1}{n^{2}}, z=\sigma+i t
$$

absolutely and uniformly converging in any finite region of the complex z-plane, for which $\sigma \geq 1+\delta, \delta>0$. If $\sigma>1$ the function is represented by the following Euler product formula

$$
\varsigma(z)=\prod_{p}\left[1-\frac{1}{p^{z}}\right]^{-1}
$$

where $p$ is all prime numbers. $\varsigma(z)$ was firstly introduced by Euler [1] in 1737, who decomposed it to the Euler product formula (2). Chebyshev [2], studying the law of prime numbers distribution, had considered this function. However, the most pro- 
found properties of the function $\varsigma(z)$ had only been discovered later, when the function had been considered as a function of a complex variable. In 1876 Riemann [3] was the first who showed that:

$\varsigma(z)$ allows analytical continuation on the whole $z$-plane in the following form

$$
\pi^{-z / 2} \Gamma(z / 2) \varsigma(z)=1 /(z(z-1))+\int_{1}^{+\infty}\left(x^{s / 2}-1+x^{(1-s) / 2}-1\right) \theta(x) \mathrm{d} x .
$$

where $\Gamma(z)$-gamma function.

$$
\theta(x)=\sum_{n=1}^{\infty} \exp \left(-\pi n^{2} x\right) \text {. }
$$

$\varsigma(z)$ is a regular function for all values of $z$, except $z=1$, where it has a simple pole with a deduction equal to 1 , and satisfies the following functional equation

$$
\pi^{-z / 2} \Gamma(z / 2) \varsigma(z)=\pi^{-(1-s) / 2} \Gamma((1-z) / 2) \varsigma(1-z)
$$

This equation is called the Riemann's functional equation.

The Riemann's zeta function is the most important subject of study and has a plenty of interesting generalizations. The role of zeta functions in the Number Theory is very significant, and is connected to various fundamental functions in the Number Theory as Mobius function, Liouville function, the function of quantity of number divisors, and the function of quantity of prime number divisors. The detailed theory of zeta functions is showed in [4]. The zeta function spreads to various disciplines and now the function is mostly applied in quantum statistical mechanics and quantum theory of pole [5] [6] [7]. Riemann's zeta function is often introduced in the formulas of quantum statistics. A well-known example is the Stefan-Boltzman law of a black body's radiation. The given aspects of the zeta function reveal global necessity of its further investigation.

The most significant contribution to the study of zeta functions is found in the results obtained by Muntz [8].

Muntz generalized all the results from the studies of zeta functions' analytical properties. He noticed that all the properties can be integrated in one theory, which is called the Muntz theorem for zeta functions.

Our goal is to use this theorem on the analogs of zeta functions. We are interested in the analytical properties of the following generalizations of zeta functions:

$$
\begin{aligned}
& P(z)=\sum_{p} \frac{1}{p^{z}} \\
& P_{m}(z)=\sum_{p \leq m} \frac{1}{p^{z}} \\
& P^{m}(z)=\sum_{p>m} \frac{1}{p^{z}} \\
& \varsigma_{p}^{m}(z)=\varsigma(z)-P^{m}(z)
\end{aligned}
$$

where $p$ are prime numbers. The forms of the given function (5)-(8) allow assuming that they possess the same properties as the zeta function (1), but it is not quite obvious, considering 


$$
\ln (\varsigma(z))=\sum_{n=1}^{\infty} P(n z) / n .
$$

we see the necessity of analyzing (5)-(8) functions for a deeper understanding of the properties of zeta functions.

\section{Results}

These are the well-known results obtained by Muntz for the zeta function.

Theorem 1. Let the function $F(x)$ be limited on every finite interval and have an order $x^{-\alpha}, \alpha>1, \dot{F}(x)$ is continuous and limited on every finite interval and has an order $x^{-\beta}, \beta>1$, then this equation holds

$$
\varsigma(z) \int_{0}^{+\infty} x^{z-1} \sum_{n=1}^{\infty} F(n x) \mathrm{d} x=\int_{0}^{+\infty} x^{z-1}\left[\sum_{n=1}^{\infty} F(n x)-\frac{1}{x} \int_{0}^{+\infty} F(v) \mathrm{d} v\right] \mathrm{d} x
$$

Let $N$ be the set of all natural numbers and $N P_{m}$ - the set of all prime numbers greater than $\mathrm{m}, N_{p}^{m}=N \backslash N P_{m}$-the set of all natural numbers without the prime numbers greater than $m$.

Below we will always let $m>3$, this limitation is introduced only to simplify the calculations. Considering all the information above let us rewrite

$$
\varsigma_{p}^{m}(z)=\sum_{n \in N_{p}^{m}} \frac{1}{n^{z}} .
$$

For the function $\varsigma_{p}^{m}(z)=\varsigma(z)-P^{m}(z)$, let us apply the results obtained by Muntz for the zeta function representation. With the help of the given definitions we formulate the analog of Muntz theorem.

Theorem 2. Let the function $F(x)$ be limited on every finite interval and have an or$\operatorname{der} x^{-\alpha}, \alpha>1$,

$\dot{F}(x)$ is continuous and limited on every finite interval and has an order $x^{-\beta}, \beta>1, F() \geq 0$, then the following equation holds for the function $\varsigma_{p}^{m}(z)$ Muntz formula is true.

$$
\begin{aligned}
& S_{p}^{m}(z) \int_{0}^{+\infty} x^{z-1} F(x) \mathrm{d} x=\int_{0}^{+\infty} x^{z-1}\left[\sum_{n \in N_{p}^{m}} F(n x)-\frac{1}{x} \int_{0}^{+\infty} F(v) \mathrm{d} v+\frac{\mu(0)}{x}\right] \mathrm{d} x \\
& \mu(x)=\frac{1}{2} \sum_{p>m_{x}} \int_{(p-1)}^{x(p+1)} F(v) \mathrm{d} v
\end{aligned}
$$

PROOF: According to the theorem conditions we have

$$
\int_{0}^{+\infty} x^{z-1} \sum_{n \in N_{p}^{m}} F(n x) \mathrm{d} x=\sum_{n \in N_{p}^{m}} \int_{0}^{+\infty} x^{z-1} F(n x) \mathrm{d} x
$$

After the substitution of variables $n x=y$ we can rewrite

$$
\sum_{n \in N_{p}^{m}} \int_{0}^{+\infty} x^{z-1} F(n x) \mathrm{d} x=\sum_{n \in N_{p}^{m}} \frac{1}{n^{z}} \int_{0}^{+\infty} y^{z-1} F(y) \mathrm{d} y=\varsigma_{p}^{m}(z) \int_{0}^{+\infty} x^{z-1} F(x) \mathrm{d} x
$$

The last steps are true and result from the theorem conditions and Weierstrass theo- 
rem of uniform convergence of improper integrals. Let us introduce the functions

$$
\begin{aligned}
& g(x)=[x],[x] \in N_{p}^{m},[x]+1 \in N_{p}^{m} \\
& g(x)=[x]+(x-[x]) / 2,[x] \in N_{p}^{m},[x]+1 \in N P_{m} \\
& g(x)=[x]+(x-[x]) / 2+1 / 2,[x] \in N P_{m},[x]+1 \in N_{p}^{m}
\end{aligned}
$$

According to the theorem conditions we have

$$
\sum_{n \in N_{p}^{m}} F(n x) \mathrm{d} x-\int_{0}^{+\infty} F(u x) \mathrm{d} u=x \int_{0}^{+\infty} \dot{F}(u x)(u-g(u)) \mathrm{d} u-\frac{1}{2} \sum_{p>m} \int_{p-1}^{p+1} F(x u) \mathrm{d} u
$$

Applying the theorem conditions we have

$$
\sum_{n \in N_{p}^{m}} F(n x) \mathrm{d} x-\int_{0}^{+\infty} F(u x) \mathrm{d} u=x \int_{0}^{1 / x} O(1) \mathrm{d} u+\int_{1 / x}^{\infty} O\left((u x)^{-\beta}\right) \mathrm{d} u-\frac{1}{2} \sum_{p>m} \int_{p-1}^{p+1} F(x u) \mathrm{d} u
$$

Substituting the variablles of the last part

$$
\sum_{n \in N_{p}^{m}} F(n x) \mathrm{d} x-\frac{1}{x} \int_{0}^{+\infty} F(v) \mathrm{d} v=x \int_{0}^{1 / x} O(1) \mathrm{d} u+\int_{1 / x}^{\infty} O\left((u x)^{-\beta}\right) \mathrm{d} u-\frac{1}{2} \frac{1}{x} \sum_{p>m_{x}(p-1)}^{x(p+1)} \int_{(2)} F(v) \mathrm{d} v(20)
$$

Calculating we obtain the following

$$
x \int_{1 / x}^{\infty} O\left((u x)^{-\beta}\right) \mathrm{d} u=C \frac{1}{1-\beta}
$$

According to the result above we obtain

$$
\sum_{n \in N_{p}^{m}} F(n x) \mathrm{d} x-\frac{1}{x} \int_{0}^{+\infty} F(v) \mathrm{d} v=x \int_{0}^{1 / x} O(1) \mathrm{d} u+\int_{1 / x}^{\infty} O\left((u x)^{-\beta}\right) \mathrm{d} u-\frac{1}{x} \mu(x)
$$

Using the properties of defined integrals and subintegral function positivity, we have

$$
\begin{aligned}
& |\mu(x)| \leq \int_{0}^{\infty}|F(v)| \mathrm{d} v \\
& \mu(x 1) \leq \mu(x 2), x 1 \leq x 2
\end{aligned}
$$

From the result above it follows that

$$
\sum_{n \in N_{p}^{m}} F(n x)-\frac{1}{x} \int_{0}^{+\infty} F(v) \mathrm{d} v=x \int_{0}^{1 / x} O(1) \mathrm{d} u+\int_{1 / x}^{\infty} O\left((u x)^{-\beta}\right) \mathrm{d} u-\frac{1}{x} \mu(0)-\frac{(\mu(x)-\mu(0))}{x}
$$

According to the Muntz theorem, we have

$$
\int_{0}^{+\infty} x^{z-1} \sum_{n \in N_{p}} F(n x) \mathrm{d} x=\int_{0}^{1} x^{z-1} \sum_{n \in N_{p}^{m}}\left[F(n x)-\frac{C}{x}\right] \mathrm{d} x+\frac{C}{z-1}+\int_{1}^{+\infty} x^{z-1} \sum_{n \in N_{p}^{m}} F(n x) \mathrm{d} x
$$

Finally, after the substitution of variables we have

$$
S_{p}^{m}(z) \int_{0}^{+\infty} x^{z-1} F(x) \mathrm{d} x=\int_{0}^{+\infty} x^{z-1}\left[\sum_{n \in N_{p}^{m}} F(n x)-\frac{1}{x} \int_{0}^{+\infty} F(v) \mathrm{d} v+\frac{\mu(0)}{x}\right] \mathrm{d} x
$$


From the last equation we obtain the Muntz formula. From which we have the regularity of the function $\varsigma_{p}^{m}(z)$ as z satisfied $1 / 2<\operatorname{Re}(z)<1$.

Theorem 3. The Riemann's function has nontrivial zeros only on the line $\operatorname{Re}(z)=1 / 2$;

PROOF: For $R 2(z)=\sum_{m=2}^{\infty} P(m z) / m$, we have

$$
|R 2(z)|=\left|\sum_{m=2}^{\infty} P(m z) / m\right| \leq \sum_{m=2}^{\infty}|P(m z) / m| \leq C_{\delta} \sum_{m=2}^{\infty}\left|-2^{m \delta} / m\right|<C C_{\delta}<\infty
$$

Applying the Muntz formula from the theorem 2

$$
\begin{aligned}
\ln (\varsigma(z)) & =P(z)+\sum_{m=2}^{\infty} P(m z) / m \\
& =P(z)+R 2(z)=\varsigma(z)-\varsigma_{p}^{m}(z)(z)-P_{m}(z)+R 2(z)
\end{aligned}
$$

estimating by the module

$$
|\ln (\varsigma(z))| \leq|\varsigma(z)|+\left|\varsigma_{p}^{m}(z)\right|+|R 2(z)|+\left|P_{m}(z)\right| .
$$

Estimating the zeta function, potentiating, we obtain

$$
\left.\left|\left(\varsigma(z)|\geq \exp [-\mid \varsigma(z))|-\mid \varsigma_{p}^{m}(z)\right)\right|-|R 2(z)|-\left|P_{m}(z)\right|\right]
$$

According to the theorem $1|\varsigma(z)|$ limited for $z$ from the following multitude

$$
(z,|z|<R,|z|>1+\delta, \delta>0)
$$

similarly, applying the theorem 2 for $\left.\mid \varsigma_{p}^{m}(z)\right) \mid$ we obtain its limitation in the same multitude. For the function $|R 2(z)|$ we have a limitation for all $z$, belonging to the half-plane $\operatorname{Re}(z)>1 / 2+1 / R$. Similarly, applying the theorem 2 for $\left|\varsigma_{p}^{m}(z)\right|$ we obtain its limitation in the same multitude and finally we obtain:

$$
|\varsigma(z)| \geq \exp \left[-C_{R}\right], \operatorname{Re}(z)>1 / 2+1 / R,|z|<R,|z|>1+\delta, \delta>0
$$

These estimations for $|P(z)|,|R 2(z)|,\left|P_{m}(z)\right|$ prove that zate function does not have zeros on the half-plane $\operatorname{Re}(z)>1 / 2+1 / R$ due to the integral representation (3) these results are projected on the half-plane $\operatorname{Re}(z)<1 / 2$ for the case of nontrivial zeros. The Riemann's hypothesis is proved.

\section{Conclusion}

In this work we obtained the estimation of the Riemann's zeta function logarithm outside of the line $\operatorname{Re}(z)=1 / 2$ and outside of the pole $z=1$. This work accomplishes all the works of the greatest mathematicians, applying their immense achievements in this field. Without their effort we could not even attempt to solve the problem.

\section{Acknowledgements}

The author thanks S.N. Baibekov for introducing the prime numbers to the problematics in the collective article [9]. Without this the work would be impossible. 


\section{References}

[1] Euler, L. (1988) Introduction to Analysis of the Infinite. Springer-Verlag, Berlin. https:/doi.org/10.1007/978-1-4612-1021-4

[2] Chebyshev, P.L. (1946) Selected Mathematical Works. Gostekhizdat, Moscow. [In Russian]

[3] Riemann, G.F.B. (1972) On the Number of Prime Numbers Less than a Given Quantity. Chelsea, New York.

[4] Titchmarsh, E.C. (1986) The Theory of the Riemann Zeta Function. 2nd Revised (HeathBrown) Edition, Oxford University Press, Oxford.

[5] Ray, D. and Singer, I.M. (1971) R-Torsion and the Laplacian on Riemannian Manifolds. Advances in Mathematics, 7, 145-210. https:/doi.org/10.1016/0001-8708(71)90045-4

[6] Bost, J.-B. (1987) Fibres determinants, determinants regularises et measures sur les espaces de modules des courbes complexes. Séminaire Bourbaki, 152-153, 113-149.

[7] Kawagoe, K., Wakayama, M. and Yamasaki, Y. (2008) The q-Analogues of the Riemann zeta, Dirichlet L-Functions, and a Crystal Zeta-Function. Forum Mathematicum, 20, 126. https:/doi.org/10.1515/FORUM.2008.001

[8] Müntz, Ch.H. (1992) Beziehungen der Riemannschen $\zeta$-Funktion zu willkurlichen reellen Funktionen. Matematisk Tidsskrift. B, 39-47.

[9] Baibekov, S.N. and Durmagambetov. A.A. (2016) Infinitely Many Twin Primes. arXiv:1609.04646 [math.GM]

Submit or recommend next manuscript to SCIRP and we will provide best service for you:

Accepting pre-submission inquiries through Email, Facebook, LinkedIn, Twitter, etc. A wide selection of journals (inclusive of 9 subjects, more than 200 journals)

Providing 24-hour high-quality service

User-friendly online submission system

Fair and swift peer-review system

Efficient typesetting and proofreading procedure

Display of the result of downloads and visits, as well as the number of cited articles Maximum dissemination of your research work

Submit your manuscript at: http://papersubmission.scirp.org/

Or contact apm@scirp.org 